\title{
Adaptive optics designs for an infrared multi-object spectrograph on TMT
}

Donald Gavel, Brian Bauman, Richard Dekany, Matthew Britton, David Andersen

Donald Gavel, Brian Bauman, Richard Dekany, Matthew Britton, David Andersen, "Adaptive optics designs for an infrared multi-object spectrograph on TMT," Proc. SPIE 6272, Advances in Adaptive Optics II, 62720R (27 June 2006); doi: $10.1117 / 12.672514$

Event: SPIE Astronomical Telescopes + Instrumentation, 2006, Orlando, Florida , United States 


\title{
Adaptive Optics Designs for an Infrared Multi-Object Spectrograph on TMT
}

\author{
Donald Gavel $^{*}$, Brian Bauman $^{\mathrm{b}}$, Richard Dekany ${ }^{\mathrm{c}}$, Matthew Britton ${ }^{\mathrm{c}}$, and David Anderson ${ }^{\mathrm{d}}$ \\ ${ }^{a} U C O / L i c k$ Observatory, University of California, Santa Cruz, CA 95064 \\ ${ }^{\mathrm{b}}$ Lawrence Livermore National Laboratory, Livermore, CA 94550 \\ ${ }^{\mathrm{c}}$ Caltech Optical Observatories, Caltech, Pasadena CA \\ ${ }^{\mathrm{d}}$ Herzberg Institute of Astrophysics, Victoria, Canada
}

\begin{abstract}
The Thirty Meter Telescope (TMT), the next generation giant segmented mirror telescope, will have unprecedented astronomical science capability. Since science productivity is greatly enhanced through the use of adaptive optics, the TMT science team has decided that adaptive optics should be implanted on all the IR instruments. We present the results of a feasibility study for the adaptive optics systems on the infrared multi-object spectrograph, IRMOS and report on the design concepts and architectural options. The IRMOS instrument is intended to produce integral field spectra of up to 20 objects distributed over a 5 arcminute field of regard. The IRMOS adaptive optics design is unique in that it will use multiple laser guidestars to reconstruct the atmospheric volume tomographically, then apply AO correction for each science direction independently. Such a scheme is made technically feasible and cost effective through the use of micro-electromechanical system (MEMS) deformable mirrors.
\end{abstract}

Keywords: Astronomical Adaptive Optics, Multi-object Spectrograph, Deformable Mirrors

\section{INTRODUCTION}

Astronomical adaptive optics systems have been successfully applied to high resolution narrow field imaging and spectroscopy. In these instruments, the corrected field of view is limited by the isoplanatic angle, the angle over which a single wavefront phase correction for the atmospheric aberrations is coherent at the science wavelength. However, the isoplanatic angle is usually much smaller than the telescope's field of view, making the use of the telescope inefficient. We introduce here a new concept, Multiple Object Adaptive Optics (MOAO), designed to make AO observing more efficient by multiplexing the AO observations over the field. MOAO uses separate wavefront correctors for each of several objects in the field.

The Thirty Meter Telescope (TMT) has commissioned a feasibility study for the Infrared Multiple Object Spectrograph (IRMOS) instrument which will use the MOAO concept to produce high resolution images of up to 20 objects on a 5 arcminute diameter field. Separate integral field units (IFUs) will slice up these fields to produce spectral data cubes of each object, with each subfield being a 40x40 grid of 50 milliarcsecond spatial elements and the spectrograph operating at up to $\mathrm{R}=4000$. The TMT science requirements for the spectrograph and $\mathrm{AO}$ system are summarized in Table 1.

A diagram of the generic MOAO architectural concept compared to other adaptive optics architectures is shown in Figure 1. To sense the wavefront needed for AO correction a number of laser guide stars and natural tip/tilt stars are used as beacons to probe the volumetric distribution of the atmospheric index of refraction. The process is analogous to that used in medical tomography, where ray sources and detectors outside of the body are used to image volumes within it. Once the volume of index deltas is determined, the control computer performs line integrals through it from science objects to telescope aperture, the result being the proper wavefront correction for those objects. Each spectrograph arm has, in front of it and at a pupil, a deformable mirror for placing the wavefront corrections. Then the light is focused at an f-number appropriate for an IFU field slicer and the rest of the spectrograph.

*gavel@ucolick.org; phone 831-459-5464; lao.ucolick.org

Advances in Adaptive Optics II, edited by Brent L. Ellerbroek, Domenico Bonaccini Calia, Proc. of SPIE Vol. 6272, 62720R, (2006) · 0277-786X/06/\$15 · doi: 10.1117/12.672514 
Table 1. SRD requirements for IRMOS/MOAO

\begin{tabular}{|l|l|}
\hline Wavelength range & 0.6 to 2.5 microns \\
\hline Field of View & $1-5$ arcsec AO corrected channel \\
\hline Field of Regard & 20 square-arcmin (up to 20 channels in this field) \\
\hline Image/Wavefront quality & $\begin{array}{l}50 \% \text { of the flux from a point source at } \lambda=1 \mu \text { into a } 0.05 \\
\text { arcsec square }\end{array}$ \\
\hline Sky coverage & $>90 \%$ at the Galactic poles \\
\hline Background & $<15 \%$ over natural sky+telescope \\
\hline
\end{tabular}

MOAO has a number of advantages over other wide field adaptive optics schemes, perhaps the most important advantage being the elimination of anisoplanatic error. In single deformable mirror AO systems, anisoplanatic error arises since the wavefront correction is good at only one point in the field, the direction of the guide star. In multiple conjugate $\mathrm{AO}(\mathrm{MCAO})$ systems, there is a generalized isoplanatic error due to approximating the atmospheric volume as a series of discrete layers ${ }^{1}$. In MOAO, each science object has its own dedicated deformable mirror with an optimized correction determined by line integral through the tomographically measured volume of atmosphere. A second advantage is the simplicity of the optics in the adaptive optics relay, since each relay is required only to pass a narrow field, the wide-field aberrations that occur in MCAO designs is not an issue. This leads to a third advantage, that since the field of view for each MOAO deformable mirror is small, the Lagrange invariant is small and a small aperture deformable mirror can be used. The small deformable mirrors we have in mind are micro-electro-mechanical systems (MEMS) with apertures of 25-40 mm. MEMS have a tremendous cost scaling advantage over traditional large aperture deformable mirror technology. The small size of the DM also drives down the overall size of the AO system, making the individual spectrograph arms compact and able to be placed at high density in the telescope's focal plane.

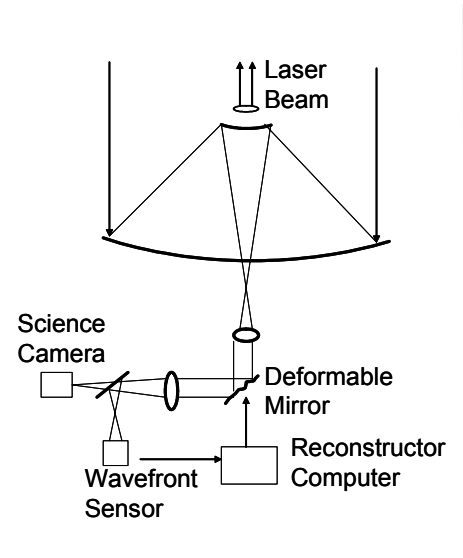

a

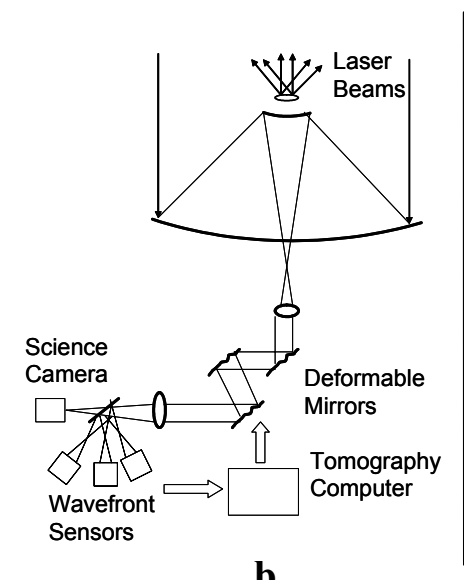

b

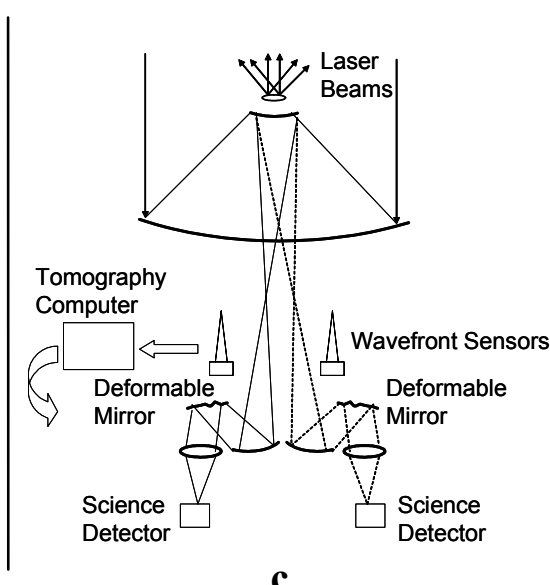

c

Figure 1. Configurations of astronomical adaptive optics systems: a) single conjugate; b) multiple conjugate; c) multiple object.

The MOAO concept is not without disadvantages and technical challenges. The control is open-loop, since the wavefront measurement is done with light that has not reflected off of the deformable mirrors. This requires extraordinarily high linearity and accuracy of the wavefront sensors over a high dynamic range (up to 20 microns of phase measured to an accuracy of about 10 nanometers). It also requires that the deformable mirrors go to where they are commanded to go, without benefit of optical feedback from the guide stars. Both of these issues have tenable solutions which we will outline in this paper.

\section{MOAO ERROR BUDGET}

The multiple object adaptive optics system has the following fundamental error budget terms: 
Tomography error is the error in measuring the volume of atmospheric turbulence given a constellation of guide stars. The error is expressed as a wavefront error that depends on field angle after integrating through the volume at that field angle. For this error it is assumed that guide stars are infinitely bright and that the tomographic reconstruction has infinite resolution in all three dimensions (pupil $u, v$ plane and altitude, $z$ ). Limiting case scaling laws for tomography error in the infinite aperture and plane wave case are derived in Tokovinin and Viard, $2001^{2}$ and further studied for the case of finite apertures and cone beams in Gavel, $2004^{3}$. A reasonable scaling law for tomographic wavefront residual error is:

$$
\sigma^{2}=\Theta^{5 / 3} r_{0}^{-5 / 3} \delta_{K}^{5 / 3} \varepsilon(\theta)
$$

where $\sigma$ is the standard deviation of the wavefront, in radians, $\Theta$ is the average separation of guidestars in the constellation, $r_{0}$ is the Fried parameter and $\delta_{K}$ is the equivalent layer thickness, which depends on the $C_{\mathrm{n}}{ }^{2}$ profile. Monte-carlo simulations of multiple LGS tomography can determine reasonable values for this number. For the nominal, Cerro-Pachon $C_{\mathrm{n}}{ }^{2}$ profile used in this study, the equivalent layer thickness is about 900 meters. The $\varepsilon(\theta)$ factor is a field-dependent term that is nominally one at the field location of any guide star and slightly larger than one in between guide stars.

Fitting error depends on the number of degrees of freedom in the deformable mirror. It is given by

$$
\sigma^{2}=\mu\left(d / r_{0}\right)^{5 / 3}
$$

where $d$ is the subaperture diameter and $\mu$ is a parameter that depends on the type of deformable mirror. For continuous face sheets, $\mu$ is approximately 0.3 .

Bandwidth error due to the finite temporal bandwidth of the real-time controller is given by

$$
\sigma^{2}=\left(f_{g} / f_{c}\right)^{5 / 3}
$$

where $f_{g}$ is the Greenwood frequency, which is proportional to the wind velocity of the turbulent layers, and $f_{c}$ is the closed loop bandwidth, which is usually about a factor of 10 lower than the control loop sample rate. This formula is reasonably accurate even for open loop systems $-f_{c}$ is simply interpreted as a control cutoff frequency, which, even in open loop is about $1 / 10$ the sample rate.

Measurement signal-to-noise error, in radians of phase at the science wavelength $\lambda$, is approximately

$$
\sigma=\eta(d / \pi \lambda)\left(\sigma_{\text {spot }} / S N R\right)
$$

where $\sigma_{\text {spot }}$ is the apparent size of the Hartmann spot on the sky, SNR is the signal-to-noise in the wavefront sensor slope measusrement, $\lambda$ is the science wavelength, $d$ is the sbuaperture diameter, and $\eta$ is the noise propagator, which is a factor that is on the order of 1.5 for the 10,000 degree of freedom MOAO system.

Tip/Tilt error. A separate study by Richard Clare of the TMT project office has led to the conclusion that tip/tilt errors using 3 natural guidestars sensed in $\mathrm{R}$ band will lead to on the order of 15 milliarcseconds of random tip/tilt error. This basically blurs the core of the PSF out to 15 mas but has almost zero effect on ensquared energy inside the 50 mas IFU spectrograph pixel.

Focus error. The focus term of the laser guide star measured wavefront is sensitive to variations in height of the Sodium layer. The depth of field of the TMT at $90 \mathrm{~km}$ is about 9 meters, therefore Sodium layer variations on the order of 9 meters, about 1/1000 of the layer's nominal thickness, would put an unacceptable amount of wavefront error on the AO correction. To avoid this error, a separate measure of focus using a natural guide star is required. The temporal power spectrum of the variations will determine how often this normalizing measurement needs to be made. Lidar measurements ${ }^{4}$ show the Sodium height fluctuates by kilometers on 5 minute time scales. The spectrum falls off with a roughly -1.6 power law in height variance $\left(\mathrm{m}^{\wedge} 2 / \mathrm{Hz}\right)$ in the range from $10^{-4.5}$ to $10^{-2.5} \mathrm{~Hz}$. However, the shortest time scale of these measurements is on the order of 5 minutes. If we extrapolate this spectrum, by decades, to the 1000 $\mathrm{Hz}$ real-time control scale this data would indicate that the LGS focus component is not at all useful for wavefront reconstruction and focus sensing of a natural star in real time is required. 
Recent measurements performed at the Lick Observatory have provided Sodium layer height fluctuation data down to time scales of one second. On 19 November, 2005, we viewed the Laser guide star from another telescope, about 600 meters west of the one projecting the laser, and collected focal plane images of the sodium layer fluorescence. The angle was such that we could resolve the layer into about 100 bins, or about 100 meters. Then, by drift-scanning in a direction perpendicular to the laser track, the CCD could resolve Sodium layer variations on a one second time scale.
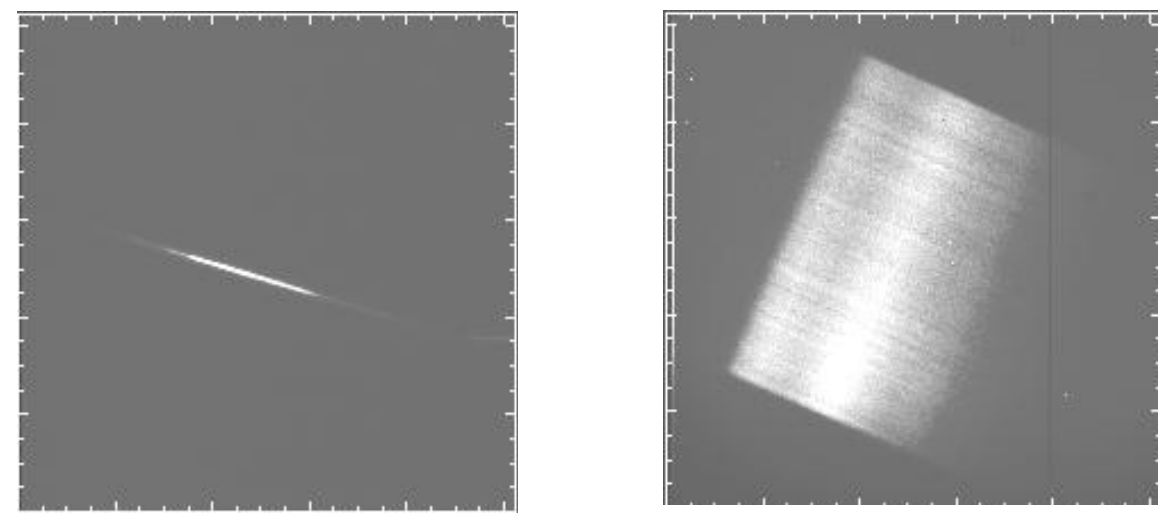

Figure 2. Left: picture of the streak of sodium return from the sodium guide star projected from the 3 meter Shane telescope, as seen from the 1 meter Nickel telescope, about $600 \mathrm{~m}$ to the west. Right: drift-scan image; time is resolved in the direction orthogonal to the streak.
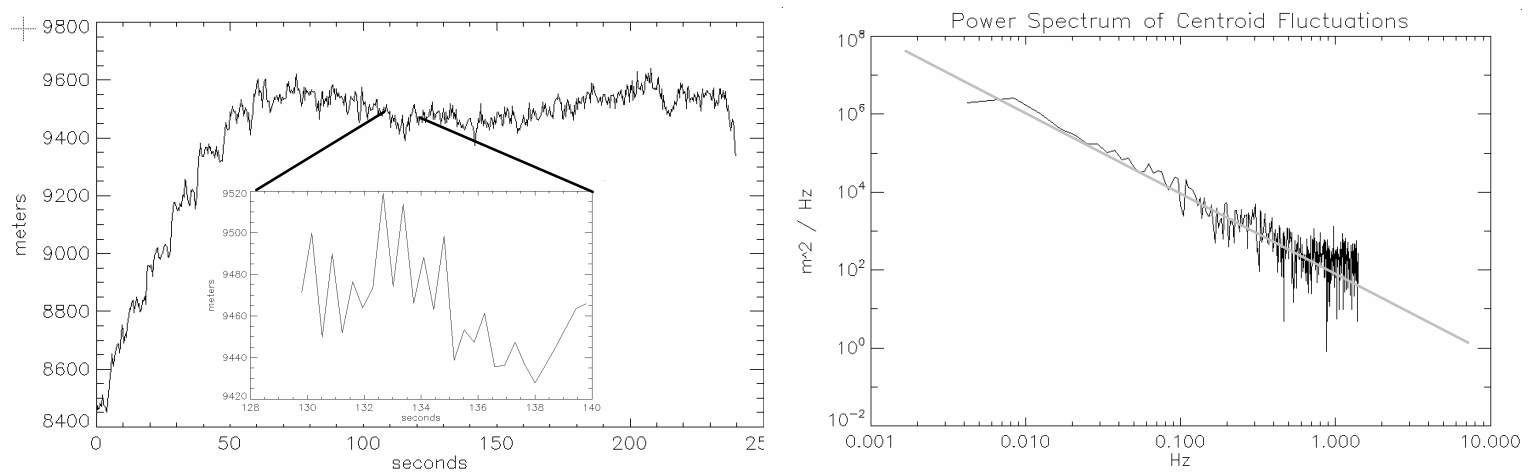

Figure 3. Typical plot of sodium centroid of height fluctuation (left) and power spectrum (right)

The data are consistent with a -2.0 power law in height variance $\left(\mathrm{m}^{\wedge} 2 / \mathrm{Hz}\right)$ in the 0.01 to $1.0 \mathrm{~Hz}$ range. 20 to 40 meter excursions occur at one second time scales, so, by extrapolation we might conclude that the, say, 1 meter excursions would be occurring on a 0.01 second time scale, and focus updates should occur at about this rate. We should caution that this data set consists of one night's observations and that further data gathering will be necessary before conclusions can be made. Additional observations of the laser from the Nickel telescope will take place during laser guidestar engineering runs this year.

The preliminary IRMOS/MOAO error budget is summarized in the table below. This error budget does yet not include some of the error terms that we are still working on quantifying, such as focus error due to sodium layer height variability discussed above, and the nonlinearities in wavefront sensing which are discussed in a later section, nor does it include systematic and manufacturing tolerance error budget terms. 
Table 2. IRMOS/MOAO error budget

\begin{tabular}{|c|c|c|}
\hline \multicolumn{3}{|c|}{ Higher Order WF (nm) } \\
\hline$\overline{\text { Fitting }}$ & 77.66 & 100 actuators across DM \\
\hline Aniso & 0.00 & (on-axis) \\
\hline Tomography & 159.97 & \\
\hline Meas & 40.00 & 180 photocounts/subap/fram \\
\hline Bandwidth & 44.66 & $1 \mathrm{kHz}$ wavefront sample rate \\
\hline Total High Order & 187.66 & $\mathrm{~nm}$ \\
\hline \multicolumn{3}{|l|}{ Tip/Tilt (mas) } \\
\hline Tilt Aniso & $\overline{14}$ & 90\% sky coverage \\
\hline Meas & $<1$ & $\mathrm{~J}=21$ \\
\hline Bandwidth & $<1$ & $\mathrm{t}=0.8 \mathrm{~ms}$ \\
\hline Total Tip/Tilt & 14 & mas \\
\hline Sky Coverage & & $90 \%$ at NGP \\
\hline
\end{tabular}

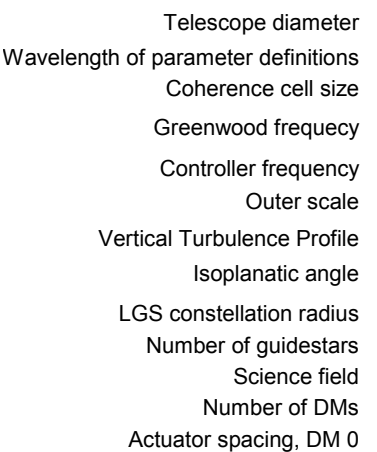

\begin{tabular}{|c|c|c|}
\hline$D$ & 30 & $\mathrm{~m}$ \\
\hline$\lambda_{0}$ & 500 & $\mathrm{~nm}$ \\
\hline$r_{0}$ & 0.15 & $\mathrm{~m}$ \\
\hline$f_{g}$ & 50 & $\mathrm{~Hz}$ \\
\hline$f_{c}$ & 100 & $\mathrm{~Hz}$ \\
\hline$L_{0}$ & $>30$ & $\mathrm{~m}$ \\
\hline$C_{n}{ }^{2}$ & Cerro Pachon & 7-layer \\
\hline$\theta_{0}$ & 2.45 & arcsec \\
\hline$\Xi$ & 120 & $\operatorname{arcsec}$ \\
\hline$N_{g s}$ & 8 & \\
\hline$\theta$ & 10 & $\operatorname{arcsec}$ \\
\hline$N_{D M}$ & 2 & \\
\hline$d$ & 0.29 & $\mathrm{~m}$ \\
\hline
\end{tabular}

\section{Laser guidestar constellation}

Tomography error rises steeply with guidestar separation angle and it is a challenge to find a constellation that will achieve reasonable measurement over the 5 arcminute MOAO field using a reasonably small number of guide stars. We have studied a number of cases where we varied the constellation configuration, size, and number of guide stars. Some of the results of this study are shown in the figures below.
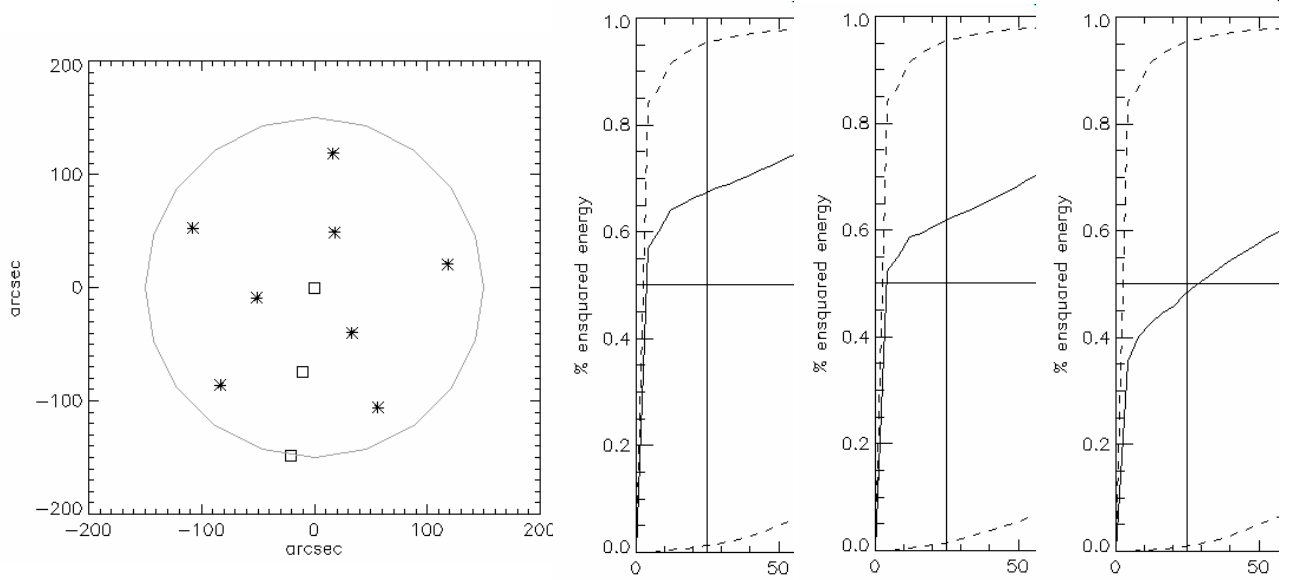

Figure 4. An 8 guidestar constellation (stars) on a 5 arcminute diameter field. The constellation outer radius is 120 arcseconds. Ensquared energy curves are shown for science objects (squares) at 0, 75, and 150 arcsecond radius.
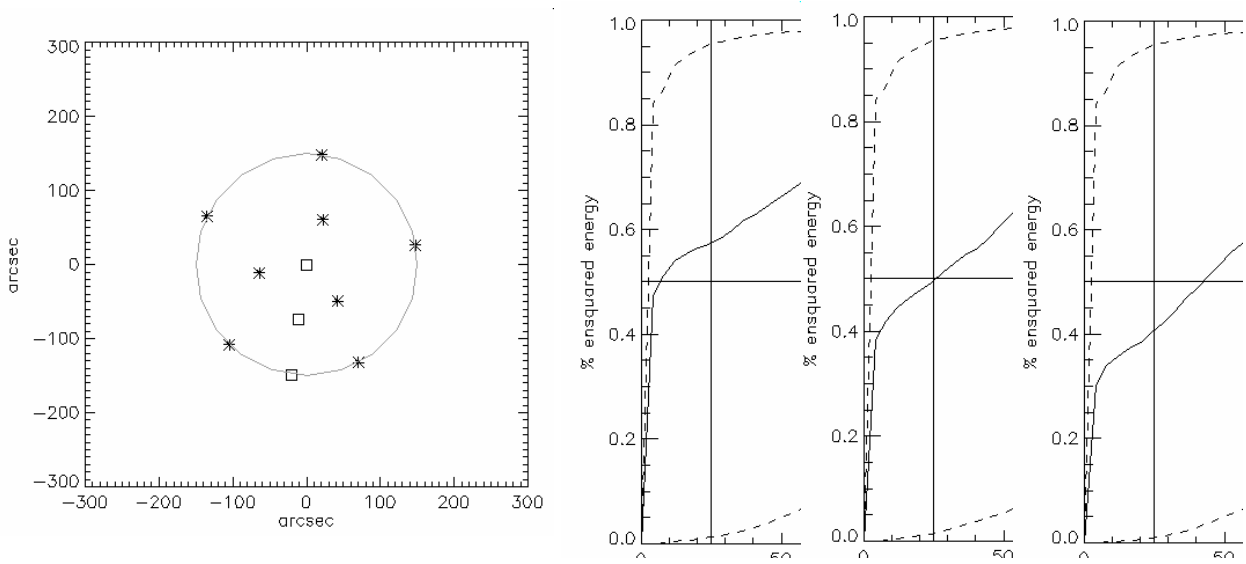

Figure 5. An 8 guidestar constellation with a 150 arcsecond constellation radius. Note that ensquared energy curves are not as good as the 120 arcsecond case, particularly at the edge of the field. 
Table 3. Summary of LGS constellation performance for a variety of constellation configurations and radii. 50 mas ensquared energy percentages at $\lambda=1$ micron science wavelength are shown for science objects at 0,75 , and 150 arcseconds radius from the center of the constellation field. The best performing constellation is highlighted.

In 50 mas square at $\lambda=1$ micron

\begin{tabular}{|c|c|c|c|c|}
\hline Constellation & $\begin{array}{c}\text { Outer diameter, } \\
\text { arcsec }\end{array}$ & \%ee 0 & \%ee 75 & \%ee 150 \\
\hline \hline $5+3$ & 150 & 0.6 & 0.5 & 0.4 \\
\hline $5+3$ & 200 & 0.42 & 0.3 & 0.3 \\
\hline $5+3$ & 120 & 0.7 & 0.62 & 0.5 \\
\hline $4+3$ & 120 & 0.65 & 0.5 & 0.4 \\
\hline $5+1$ & 120 & 0.48 & 0.4 & 0.3 \\
\hline $5+2$ & 120 & 0.65 & 0.5 & 0.35 \\
\hline $6+2$ & 120 & 0.6 & 0.6 & 0.45 \\
\hline
\end{tabular}

\section{STATUS OF MEMS DEFORMABLE MIRROR DEVELOPMENT}

The Laboratory for Adaptive Optics, in collaboration with the Center for Adaptive Optics, Gemini Observatory, and the TMT project, is pursuing the development of micro-electro-mechanical system (MEMS) technology suited for adaptive optics for large aperture telescopes. Currently, the most promising design is from the Boston Micromachines Corporation (BMC), which has shown good progress in making highly reliable, high yield, and nominally flat deformable mirrors with high actuator count. This device consists of discrete electrostatically controlled actuators each with a stiff post attached to a continuous top plate in order to exert force and therefore deflect the plate. The plate is coated with either aluminum or gold to form the reflective surface.

MEMS technology provides a number of advantages over conventional deformable mirror approaches:

- Small size - microfabrication on silicon wafers allow very small inter-actuator separation, on the order of 400 microns on the devices we are testing.

- High actuator count at reasonable cost - The marginal cost of scaling to high actuator counts is considerably lower than that for large DMs. For piezo electric actuate mirrors this cost appears to be around $\$ 1500$ per actuator including drive electronics, or about $\$ 15 \mathrm{M}$ for a 10,000 element DM. Given some rough order of magnitude estimates for the cost of fabricating wafers, packaging, bonding, and the known cost of drive electronics, we can expect costs for MEMS devices to be about $\$ 40$ per actuator, or about $\$ 400 \mathrm{~K}$ for a 10,000 actuator device.

- "Go-to" repeatability - One major advantage of an electrostatic actuation over piezoelectric actuation is the absence of hysteretic effects in the displacement to voltage response curves. This implies that the devices could be driven open loop to given surface deflections. Open-loop tests of stability and repeatability are presented below.

The low cost and small size of MEMS DMs opens up the possibility of "ubiquitous MEMS," i.e. devices sprinkled throughout the system to elegantly solve tough optical problems.

- MEMS DM in each wavefront sensor: This creates a mini closed-loop AO system in which the wavefront detector is kept near null, where its linearity properties are best. The predictable voltage response of the MEMS allows it to be used as the probe of the grosser portion of the wavefront shape, which would be added to the wavefront sensor's residuals to complete the wavefront measurement.

- MEMS in the tip/tilt sensors: Diffraction limited images of the tip/tilt stars at the sensing wavelength will yield greater tip/tilt accuracy because centroid error is proportional to the spot size and inversely proportional to square root of star intensity. The ability to adaptively correct tip/tilt stars would lead to notably higher sky coverage since dimmer tip/tilt stars could then be used. The baseline specifications for IRMOS will not require this approach, since the encircled energy requirements are relaxed enough to permit adequate tip/tilt sensing using seeing limited stars in the $\mathrm{R}$ band. This concept however could be used to advantage in more stressing AO design concepts, such as MCAO diffraction limited imaging or in an IRMOS high-Strehl "superbutton," where a diffraction limited PSF is desired in combination with good sky coverage. 
- MEMS in the laser projector: Uplink correction, i.e. correction of the laser wavefronts for the distortions they will see along the upward path through the atmosphere, will produce smaller illuminated spots in the Sodium layer. There would need to be one DM per beam, since each goes through a different atmospheric path, and the small size of the MEMS lends itself well to placement in a pupil conjugate in the laser paths ahead of the launch telescope.
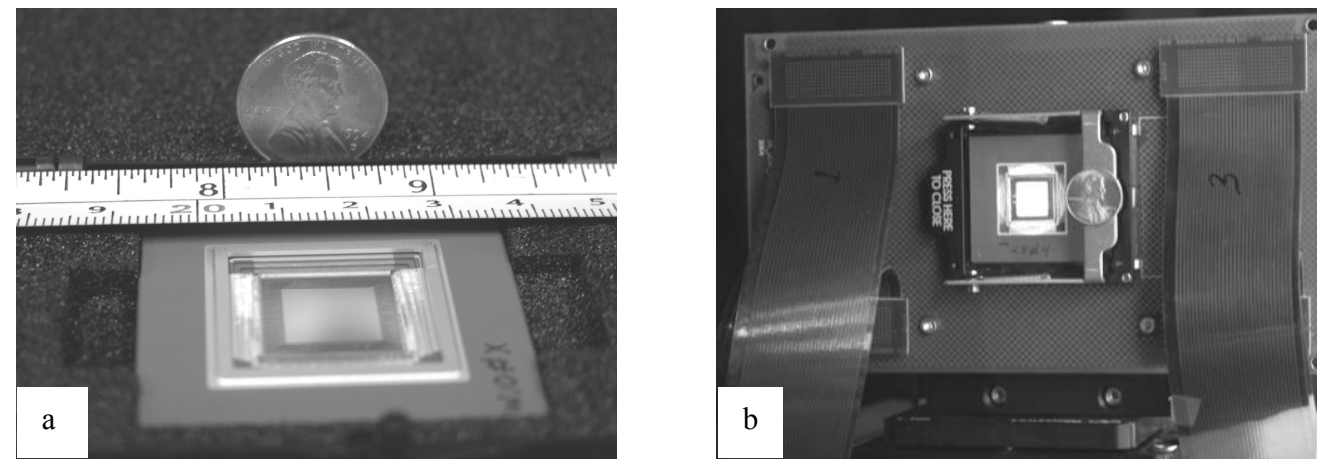

Figure 6. a) 1000 actuator Boston Micromachines (BMC) MEMS deformable mirror. This is a $32 \times 32$ actuator array at 360 microns pitch. b) MEMS mirror plugged into its electrical connector board with cabling shown. The green disk is the $532 \mathrm{~nm}$ PSDI interferometer beam.

MEMS technology is fairly new, and as such, presents a development risk.

- Limited stroke - present technology for the electrostatic actuator is at about 4 microns surface deflection, compared to the approximately 10 microns needed for the 30 meter aperture of TMT. To work around this in a design, a second low-order high stroke deformable mirror, or "woofer" mirror, must be placed in the path. Fortunately, the amplitude of the Kolmogorov atmospheric wavefront spectrum falls rapidly with spatial frequency so the majority of the stroke is concentrated at the low spatial frequencies.

- Reliability - The MEMS mirrors have been extensively field tested where conditions are similar to those found in a dome/mountaintop environment ${ }^{5}$. MEMS have also been extensively life-cycle tested in the laboratory. BMC has done billion-actuation tests of their actuator with no evidence of fatigue. However, MEMS have not yet been proven in routine astronomical telescope use and the reliability and failure rates have yet to be proven convincingly for astronomers.

- Yield - Yield relates ultimately to per device cost, and with the yield not yet known for 4000 and 10000 actuator devices we have difficulty fully understanding the cost. BMC has made considerable progress increasing the yield on their 1000 actuator devices under the earlier development contract with LAO. Similar results in yield on the higher actuator count devices will undoubtedly lead to a per unit cost that is a factor of 10 to 30 below the cost of piezo DMs.

- Vendor sources - In today's market, MEMS providers are generally small companies, often associated with the telecom industry, as opposed to large aerospace firms with considerable experience producing conventional technology DMs.

The fact that a new technology has never been used in astronomy before should certainly not be a deterrent to astronomers pioneering its use. As an example, the application of CCDs as astronomical detectors in the 1970's provides a precedent where tremendous breakthroughs in science can be achieved using new technologies in new instruments.

\section{Results of Laboratory Testing of $1 K$ Actuator Devices}

A number of $1 \mathrm{~K}$ actuator MEMS devices have been delivered to the Laboratory for Adaptive Optics for testing. These mirrors have a surface stroke of up to 2 microns with a $200 \mathrm{~V}$ actuation applied. We tested the devices for long stability and repeatability of surface figure using a very precise point diffraction interferometer. We also flattened the surface and measured the spatial frequency distribution of the residual as well as the resulting point spread function in the far field. ${ }^{6,7}$ 

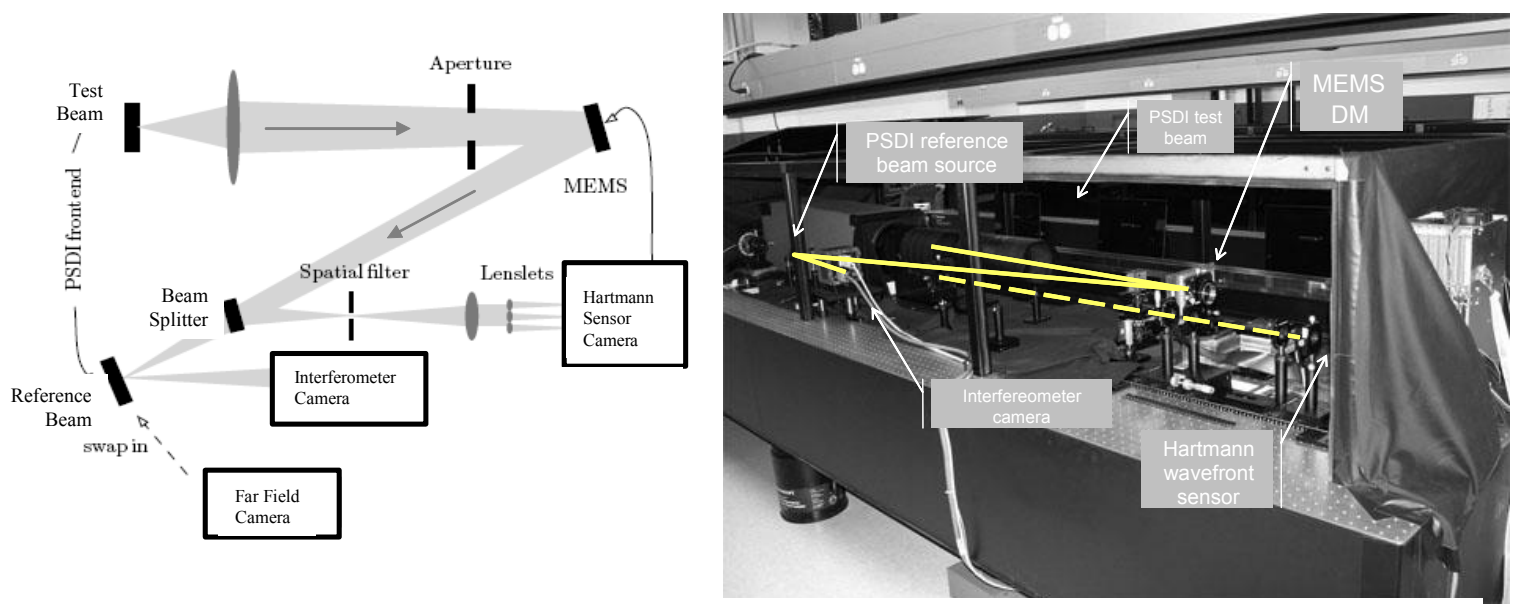

Figure 7. The MEMS device test set up in the phase-shifting diffraction interferometer (PSDI) on the LAO Extreme Adaptive Optics Testbed.

Typical MEMS mirror flattening performance is shown in Figure 8. The unpowered mirror reflected wavefront has $148.1 \mathrm{~nm}$ phase departure from flat, mostly low-order aberration as shown. After flattening, the mirror has $12.8 \mathrm{~nm}$ of wavefront error, with $0.54 \mathrm{~nm}$ located inside the Nyquist sampling spatial bandwidth of the actuators. Thus approximately $12 \mathrm{~nm}$ is attributed to "orangepeel" and other micro roughness at spatial frequencies above Nyquist. This is comparable to the best piezo actuated mirrors, where after-correction surface roughness of about $10 \mathrm{~nm}$ rms phase has been reported. $30 \mathrm{~nm}$ rms post-correction phase is typical with piezo DMs in present day astronomical AO systems.
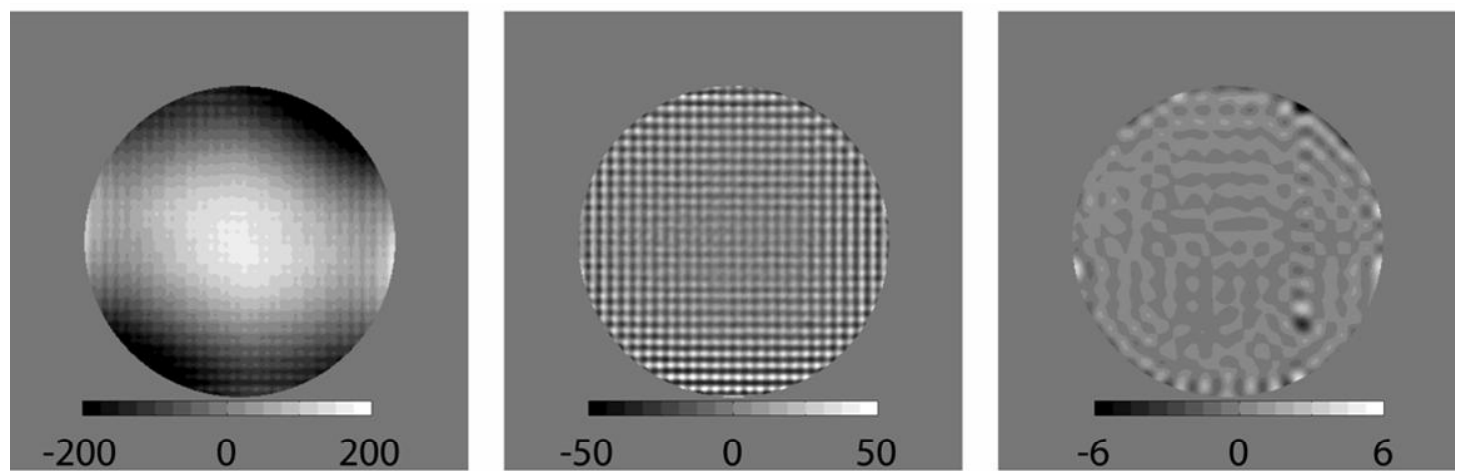

Figure 8. Results of flattening the MEMS mirror. Left: unpowered surface. Center: Flattened surface. Right: Flattened surface with the data spatially low-pass filtered with filter cutoff at the Nyquist frequency. Note the changes in scale.

Table 4. Table of MEMS flattening test results

\begin{tabular}{|l|l|l|}
\hline & Unpowered & Flattened \\
\hline Total rms wavefront error & $148.1 \mathrm{~nm}$ & $12.8 \mathrm{~nm}$ \\
\hline rms wavefront error in band & $144.1 \mathrm{~nm}$ & $0.54 \mathrm{~nm}$
\end{tabular}

In a test of long term stability we gave the MEMS a fixed set of voltage commands and took differences of interferometer-measured wavefronts over various time scales between a minute and roughly half an hour. These were compared to similar measurements using a test flat. To test go-to repeatability, we measured the surface at a given command position, then moved the MEMS to flat, then returned to the command position. This was repeated for several cycles over a time span of roughly an hour. Anecdotally, the go-to repeatability does not appear to change even over days (additional long-term tests will be performed to confirm this). Results of these tests are summarized in Table 5 . 
Table 5. Results of MEMS stability and repeatability tests.

- $96 \%$ of the actuators display quadratic voltage-displacement curves.

- Go-to capability:

$97 \%$ of the actuators tested went to a commanded shape to under $1.0 \mathrm{~nm}$ phase

$73 \%$ of the actuators tested went to a commanded shape to under $0.4 \mathrm{~nm}$ phase

- MEMS Stability:

$0.088 \mathrm{~nm}$ phase over $1.33 \mathrm{~min}$.

$0.106 \mathrm{~nm}$ phase over 8 minutes.

$0.150 \mathrm{~nm}$ phase in 38 minutes.

$62 \%$ of actuators* were stable to under $0.1 \mathrm{~nm}$ phase stddev.

$89 \%$ of actuators were stable to under $0.2 \mathrm{~nm}$ phase stddev.

$96 \%$ of actuators were stable to under $0.3 \mathrm{~nm}$ phase stddev.

*By "actuator" we mean average surface height over one interactuator spacing sized

area, centered on an actuator

- Reference flat mirror, for comparison:

$0.042 \mathrm{~nm}$ phase over $0.33 \mathrm{~min}$.

$0.116 \mathrm{~nm}$ phase over $8 \mathrm{~min}$.

$0.122 \mathrm{~nm}$ phase over $38 \mathrm{~min}$.

Instrumental error of the PSD Interferometer is on the order of $0.2 \mathrm{~nm}$ per measurement. Errors in the numbers reported above were beaten down by repeated measurements and fitting to trend lines.

\section{OPEN LOOP CONTROL}

One of the unique features of the MOAO concept is the necessity for open loop wavefront sensing and control. To accomplish this successfully, both the wavefront sensor and the deformable mirror must be linear (strictly speaking, linearizable, perhaps with lookup tables) over the entire dynamic range of phase aberration expected in uncorrected seeing conditions. For IRMOS this means open loop sensing and control of up to 20 microns of optical path to an accuracy of roughly $100 \mathrm{~nm}$.

\section{Wavefront sensing}

The specialized Hartmann wavefront sensor design proposed by Jerry Nelson (U.C. Santa Cruz) and Jim Beletic (Rockwell Scientific) has the pixels arranged such that the radially elongated laser guide star spots are aligned along the grid of pixels. A CW laser illuminating the Sodium layer will produce an elongated spot on the detector for each Hartmann spot. To determine the wavefront slopes, the centroid of the elongated spot must be calculated given the photocounts in each pixel. As the spot deflects from its nominal position due to atmospheric seeing, there will be a pixelization error, which will manifest itself as a nonlinearity of the read out tilt motion vs true tilt motion. Representative plots of the $\mathrm{x}$ (across the elongated spot) and $\mathrm{y}$ (along the elongated spot) tilt measurement error due to nonlinearity are shown in Figure 8. It is assumed that the initial offset bias is subtracted and that the tilt readout is normalized to a slope of one at small deflections. Curves are shown for various numbers of pixels assigned to the Hartmann subaperture. The elongation chosen for these simulations, 2.56 arcsec, is the area-weighted elongation over the 30 meter aperture for a guide star at zenith. The overploted dotted line shows the probability distribution of openloop wavefront tilts over a subaperture in these seeing condition $\left(r_{0}=16 \mathrm{~cm}\right)$. 


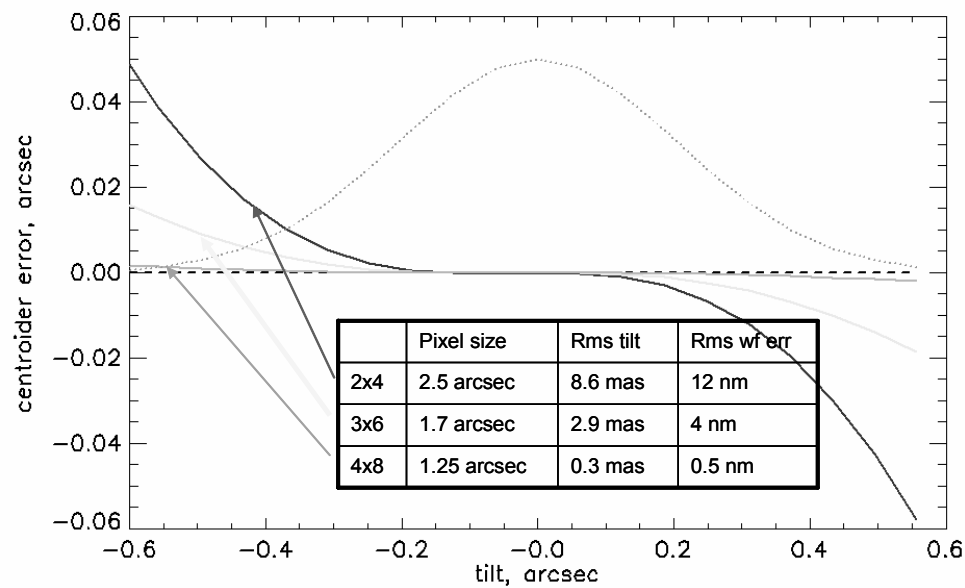

Figure 9. Hartmann tilt measurement error of an elongated LGS spot due to pixelization nonlinearity. See text for full explanation. The graph is for tilts in the direction across the elongation. Tilt errors along the elongation direction are essentially zero on this scale. The probability distribution of open loop tilts is overplotted with a dotted line. Rms tilt errors and extrapolated rms wavefront errors are summarized in the table.

The table in the graph shows rms tilt error due to the nonlinearity (averaged according to the probability distribution) and an extrapolation to the equivalent rms wavefront error over the entire aperture, assuming a noise propagator of unity in the wavefront reconstruction. This noise propagator is likely to be closer to 1.5 for a 10,000 actuator system, using Noll's formula ${ }^{8}$ for Hartmann slope to phase sensors: noise propagator $=2\left(0.0068+0.0796 \ln \left(\mathrm{N}_{\mathrm{dof}}\right)\right)$. Also, the open loop slopes, and hence the nonlinearity errors, will be systematically correlated, so the noise propagator concept, which assumes independent errors, does not strictly apply. Regardless, it is evident that the effect of nonlinearity in open loop wavefront sensing is a small, though not negligible, contribution to the error budget.

\section{Wavefront control}

MEMS deformable mirrors have the wonderful property that since the actuator forces and deflections depend solely on electrostatic attraction and the elastic deformation of silicon, the actuation is almost perfectly repeatable, i.e no hysteretic behavior is observable down to $0.1 \mathrm{~nm}$ motions. However, the surface deflection is certainly not a linear function of voltage, and plus the response of a given actuator depends on the position of neighboring actuators, also in a nonlinear manner. Thus there remains the problem of determining the voltage commands, unique though they are, that will produce a given mirror surface shape. Because of the nonlinear interactions there is no reason to believe that traditional "actuator response functions," deformation in response to unit voltage on an actuator, can be added up in linear combinations to produce a surface deformation with the accuracy that is required for open-loop control.

An alternative is to use a theoretical basis in the development of the mirror control model, using the thin plate equation and other models for actuator deflection in an electric field. Vogel and Yang ${ }^{9}$ have taken this approach to build mathematical models for the actuators and top plate.

In our more hybrid approach, the plate equation is used to determine plate forces that deflect the top surface into the desired shape and an empirical model is used for the actuators. This has an advantage over a completely theoretical model in that the actuator design in the MEMS device is usually very complicated and therefore is not easy to model as a simple electrostatic membrane. On the other hand, the hybrid approach we propose is quite amenable to laboratory calibration.

The top plate is assumed to obey the thin plate equation, given by

$$
D \nabla^{4} z(x)=f(x)=\sum_{i=1 \ldots n} f_{i}\left(x-x_{i}\right)
$$

where $z(x)$ is the displacement of the plate, $f(x)$ is the force, $D$ is the flexural rigidity, and $n$ is the number of actuators. The plate equation behaves linearly in response to forces (for small deflections that do not stretch the plate - this condition is very nearly true for MEMS top plates). At each actuator attachment to the top plate, there are three forces in effect that balance to zero net force (see Figure 10): the electrostatic attraction force which depends on applied actuator voltage, $V$, and actuator displacement, $w$; the spring return force which depends only on the actuator displacement $w$; and the bending plate's response force as exerted through the supporting post which depends on $z(x)$ through the plate equation. The three forces balance at the post: 


$$
f_{P}\left(z_{i}\right)=f_{E}\left(V_{i}, w_{i}\right)-f_{S}\left(w_{i}\right)
$$

where the subscript $i$ indicates the $i$ 'th actuator. The term on the left hand side of (6) is determined by solving the plate equation (5) given the desired surface shape. The terms on the right hand side do not interact among actuators so empirically determined nonlinearity correction tables can be used for each actuator separately to look up the voltages. There is one free parameter, $z$ - $w$, which has a single value for the whole device. It corresponds to the piston term and can be set arbitrarily to keep all actuators optimally within their dynamic range.

Figure 10. Forces and displacements in the MEMS Deformable mirror.

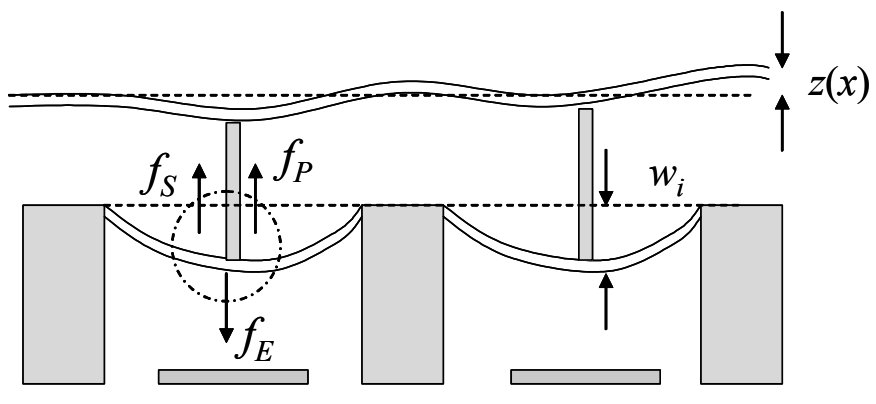

The electrostatic and spring force vs voltage curves (terms on the right hand side of (6)) can themselves be calibrated by interferometer measurements of the deflection of the top surface in response to various voltages, using the plate equation to determine the forces as well. Experiments in progress, using a laboratory interferometer and Boston Micromachines MEMS devices, are showing consistent agreement with this modeling approach and we are now starting to control the mirror to specified shapes. All of the calculations mentioned (lookup tables and solving the plate equation) can be feasibly implemented on dedicated real-time control processors.

\section{LGS WAVEFRONT SENSOR OPTICAL DESIGN}

In the IRMOS/MOAO concept, guidestar light is picked off ahead of the instrument. Figure 11 shows a layout concept with vertically mounted IRMOS enclosure and wavefront sensors. The vertical mounting simplifies the implementation of field rotation. The IRMOS instrument can rotate with the field, while the LGS wavefront sensor unit rotates independently with the guide star constellation, since this constellation might be fixed with respect to the pupil. The entire wavefront sensor assembly can be translated vertically to track sodium layer distance with zenith angle.

The ray trace begins at lower left, at the edge of the primary. A $45^{\circ}$ fold mirror reflects the beam upwards towards the dichroic. The science light transmits through the dichroic to the IRMOS buttons, which are located on a drum that rotates around a vertical axis for tracking. The LGS light reflects off the dichroic, a fold mirror, and is then collimated after focus onto a mirror located at a pupil, possibly a MEMS DM. The light is then reflected downward. A subsequent relay images the pupil onto the lenslet array in a SH WFS. The small boxes are $200 \mathrm{~mm}$ cubes and represent the WFS CCD. The WFS's are located on another vertically-oriented rotating drum. Envelope dimensions are shown. The distance from the edge of the primary to the center of the fold mirror is 1.0 meter, and the distance from the center of the fold mirror to the science instrument is 4.0 meters (cf. $0.5 \mathrm{~m}$ and $4.5 \mathrm{~m}$ dimensions above to the edges of the mirrors).

Converging laser guide star light from the telescope enters from right and is collimated by a small $25 \mathrm{~mm}$ diameter lens. The light reflects off the pupil mirrors (the diameter shown is $100 \mathrm{~mm}$ ) and then relayed via a two-element telescope to a Hartmann lenslet array. A second two-element relay then images the Shack-Hartmann dots onto the CCD, which is shown as a $100 \mathrm{~mm}$ cube.

\section{CONCLUSION}

The multiple object adaptive optics (MOAO) concept for an Infrared Multi-Object Spectrograph (IRMOS) has been under study as a premier instrument for the Thirty Meter Telescope. The adaptive optics system is made feasible and cost effective with the use of MEMS deformable mirrors in each of 20 spectrograph arms, each dedicated to a small science IFU field but deployable over the wide field. Considerable progress has been made in the laboratory to prove out the technical viability of MEMS devices as open-loop controllable deformable mirrors. 


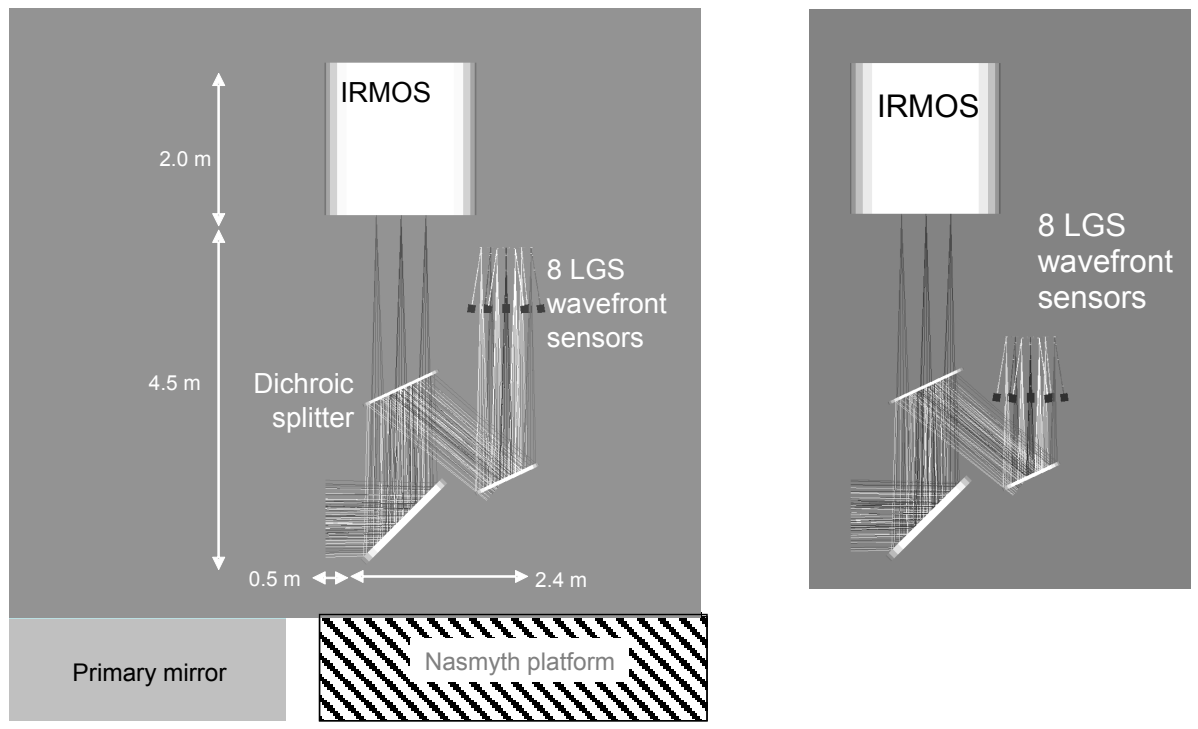

Figure 11. IRMOS with LGS wavefront sensors conjugate to $90 \mathrm{~km}$ (left) and $180 \mathrm{~km}$ (right).

\section{ACKNOWLEDGEMENTS}

This work was supported in part by the Thirty Meter Telescope project through a work-package contract in support of the feasibility study for the Infrared Multi-Object Spectrograph. This work was also supported by the Gordon and Betty Moore Foundation through their generous grant forming the Laboratory for Adaptive Optics at U.C. Santa Cruz. The theoretical tomography development work was supported by a research grant from the NSF Center for Adaptive Optics, managed by the University of California at Santa Cruz under cooperative agreement No. AST-9876783.

\section{REFERENCES}

1. Tokovinin, A., Le Louarn, M., Sarazin, M., "Isoplanatism in a multiconjugate adaptive optics system,” JOSA-A, 17, 10, pp. 1819-1827, Oct., 2000.

2. Tokovinin, A., Viard, E., "Limiting precision tomographic phase estimation," JOSA-A, 18, 4, pp 873-882, Apr. 2001.

3. Gavel, D.T., "Tomography for multiconjugate adaptive optics systems using laser guide stars," Proc. SPIE, 5490, June, 2004, pp 1356-1373.

4. Herriot, G., Herzberg Institute of Astrophysics, Private communication; based on data gathered with the Colorado State University Sodium Lidar.

5. Baker, K., Stappaerts, E., Gavel, D., Tucker, J., Silva, D., Wilks, S., Olivier, S., Olsen, J., “Adptive compensation of atmospheric turbulence utilizing an interferometric wavefront sensor and a high-resolution MEMS-based spatial light modulator," SPIE 5553, pp. 269-280, 2004.

6. Evans, J., Morzinski, K., Reza, L., Severson, S., Poyneer, L., Macintosh, B., Dillon, D., Sommargren, G., Palmer, D., Gavel, D., and Olivier, S., "Extreme adaptive optics testbed: high contrast measurements with a MEMS deformable mirror," SPIE 5905, pp. 303-310, 2005.

7. Evans, J., Macintosh, B., Poyneer, L., Morzinski, K., Severson, S., Dillon, D., Gavel, D., Reza, L., "Demonstrating sub-nm closed loop MEMS flattening," Optics Express, in press, 2006.

8. Noll, R., "Phase estimates from slope-type wave front sensors," JOSA 68, p 139, 1978.

9. Vogel C., and Yang, Q., "Modeling, Simulation, and Open Loop Control of a Continuous Facesheet MEMS deformable Mirror," JOSA-A, accepted for publication, 2006. 